Nr. $180 / 94$

A Note on Population Growth in a Crowded

STOCHASTIC ENVIRONMENT

Fred Espen Benth 


\title{
A Note On Population Growth In A Crowded Stochastic Environment
}

\author{
Fred Espen Benth \\ Department of Mathematics \\ University of Oslo \\ Box 1053 Blindern, N-0316 Oslo \\ Norway \\ $\&$ \\ Lehrstuhl für Mathematik V \\ Universität Mannheim A5 \\ D-68131 Mannheim \\ Germany \\ abstract
}

We find an explicit unique solution in the space of Kondratiev distributions, $(\mathcal{S})^{-1}$, to a stochastic differential equation modelling population growth in a crowded stochastic environment.

\section{Introduction}

In this paper we are going, to study a stochastic version of the Verhulst model for population growth,

$$
X_{t}=X_{0}+r \int_{0}^{t} X_{s} \diamond\left(N-X_{s}\right) d s+\alpha \cdot \int_{0}^{t} X_{s} \diamond\left(N-X_{s}\right) \delta B_{s}
$$

where $r, \alpha, N$ are constants, $N, r$ positive. $\delta B_{s}$ denotes the (generalized) Skorohod integral. A precise meaning of this integral will be given in the next section. We denote by $\diamond$ the Wick product.

(1) was first proposed by Lindstrøm et. al. [LØU] as a modell for population growth in a crowded stochastic environment. For deterministic initial conditions $X_{0}$, where $0 \leq$ $X_{0} \leq 1$ and $X_{0} \neq \frac{1}{2}$, they found an explicit solution to (1) using white noise methods. Their solution is a "true" stochastic variable. The case $X_{0}=\frac{1}{2}$ represents some kind of "stochastic bifurcation point", since no stochastic variable exists as a solution for this initial condition (see Lindstrøm et. al. [LØU] for their remark.) The main motivation for this paper is to give an explicit solution also for the case $X_{0}=\frac{1}{2}$. In section 4 we show that for this initial condition, we do not even have a solution in the space of Hida distributions, $(\mathcal{S})^{*}$. This suggests that the space of Kondratiev distributions, $(\mathcal{S})^{-1}$, is the natural space for this problem. Moreover, using Wick Calculus on the space of Kondratiev distributions, $(\mathcal{S})^{-1}$, we are able to find an explicit solution of $(1)$ for general initial conditions with positive expectation. Now, however, the solution is no longer a stochastic variable, but a generalized stochastic variable living in the abstract space $(\mathcal{S})^{-1}$. 


\section{Some Preliminaries}

We start by recalling some of the basic definitions and features of the white noise analysis. For a more complete account, see Hida et. al. [HKPS] and Gjessing et. al. [GHLøUZ].

As usual, let $\mathcal{S}^{\prime}\left(\mathbb{R}^{d}\right)$ denote the space of tempered distributions on $\mathbb{R}^{d}$, which is the dual of the well-known Schwartz space $\mathcal{S}\left(\mathbb{R}^{d}\right)$. By the Bochner-Minlos theorem there exists a measure $\mu$ on $\mathcal{S}^{\prime}$ such that

$$
\int_{\mathcal{S}^{\prime}} e^{i<\omega, \phi\rangle} d \mu(\omega)=e^{-\frac{1}{2}\|\phi\|^{2}}, \phi \in \mathcal{S}
$$

where $\|:\|$ is the $L^{2}\left(\mathbb{R}^{d}\right)$-norm. $\langle\cdot, \cdot\rangle$ is the dual pairing between $\mathcal{S}^{\prime}$ and $\mathcal{S}$. Let $\mathcal{B}$ denote the Borel sets on $\mathcal{S}^{\prime}$ (equipped with the weak star topology). Then the triple $\left(\mathcal{S}^{\prime}, \mathcal{B}, \mu\right)$ is called the white noise probability space.

If we define

$$
\tilde{B}_{x}(\omega):=\tilde{B}_{x_{1}, \ldots, x_{d}}(\omega):=\left\langle\omega, \mathcal{X}_{\left[0, x_{1}\right] \times \ldots \times\left[0, x_{d}\right]}(\cdot)\right\rangle
$$

then $\tilde{B}_{x}$ has an x-continuous version $B_{x}$ which becomes a $d$-parameter Brownian motion. The $d$-parameter Wiener-Ito integral of $\phi \in L^{2}$ is defined by

$$
\int_{\mathbb{R}^{d}} \phi(y) d B_{y}(\omega)=\langle\omega, \phi\rangle
$$

Of special interest will be the space $L^{2}\left(\mathcal{S}^{\prime}\left(\mathbb{R}^{d}\right), \mu\right)$, or $L^{2}(\mu)$ for short. The Wiener-Ito chaos expansion theorem says that every $F \in L^{2}(\mu)$ has the form

$$
F(\omega)=\sum_{n=0}^{\infty} \int_{\left(\mathbb{R}^{d}\right)^{n}} f_{n}(u) d B_{u}^{\otimes n}(\omega)
$$

where $f_{n} \in L^{2}\left(\mathbb{R}^{n d}\right)$ and $f_{n}$ is symmetric in its $n$ variables (in the sense that $f_{n}\left(u_{\sigma_{1}}, \ldots, u_{\sigma_{n}}\right)=$ $f_{n}\left(u_{1}, \ldots, u_{n}\right)$ for all permutations $\sigma$, where $\left.u_{i} \in \mathbb{R}^{d}\right)$. The right hand side of (2) are the multiple Ito integrals.

There is an equivalent expansion of $F \in L^{2}(\mu)$ in terms of the Hermite polynomials:

$$
h_{n}(x)=(-1)^{n} e^{\frac{x^{2}}{2}} \frac{d^{n}}{d x^{n}}\left(e^{-\frac{x^{2}}{2}}\right) ; n=0,1,2, \ldots
$$

We explain this more closely: Define the Hermite function $\xi_{n}(x)$ of order $n$ as

$$
\xi_{n}(x)=\pi^{-1 / 4}((n-1) !)^{-1 / 2} e^{-\frac{x^{2}}{2}} h_{n-1}(\sqrt{2} x)
$$

where $x^{\prime} \in \mathbb{R}, n=1,2, \ldots\left\{\left\{\xi_{n}\right\}_{n=1}^{\infty}\right.$ forms an orthonormal basis for $L^{2}(\mathbb{R})$. Therefore the family $\left\{e_{\alpha}\right\}$ of tensor products

$$
e_{\alpha}:=e_{\alpha_{1}, \ldots, \alpha_{m}}:=\xi_{\alpha_{1}} \otimes \ldots \otimes \xi_{\alpha_{d}}
$$


(where $\alpha$ denotes the multi-index $\left(\alpha_{1}, \ldots, \alpha_{d}\right)$ ) forms an orthonormal basis for $L^{2}\left(\mathbb{R}^{d}\right)$. Assume that the family of all multi-indices $\beta=\left(\beta_{1}, \ldots, \beta_{d}\right)$ is given a fixed ordering

$$
\left(\beta^{(1)}, \beta^{(2)}, \ldots, \beta^{(n)}, \ldots\right)
$$

where $\beta^{(k)}=\left(\beta_{1}^{(k)}, \ldots, \beta_{d}^{(k)}\right)$. Put

$$
e_{n}=e_{\beta^{(n)}} ; n=1,2, \ldots
$$

Let $\alpha=\left(\alpha_{1}, \ldots, \alpha_{m}\right)$ be a multi-index. It was shown by Ito that

$$
\int_{\left(\mathbb{R}^{d}\right)^{n}} e_{1}^{\widehat{\otimes} \alpha_{1}} \widehat{\otimes} \ldots \widehat{\otimes} e_{m}^{\widehat{\otimes} \alpha_{m}} d B^{\otimes n}=\prod_{j=1}^{m} h_{\alpha_{j}}\left(\theta_{j}\right)
$$

where $\theta_{j}(\omega)=\int_{\mathbb{R}^{d}} e_{j}(x) d B_{x}(\omega), n=|\alpha|$ and $\widehat{\otimes}$ denotes the symmetrized tensor product (e.g., $f \widehat{\otimes} g(x, y)=\frac{1}{2}[f(x) g(y)+f(y) g(x)]$ if $x, y \in \mathbb{R}$ and similarly for more than two variables). If we define, for each multiindex $\alpha=\left(\alpha_{1}, \ldots, \alpha_{m}\right)$,

$$
H_{\alpha}(\omega)=\prod_{j=1}^{m} h_{\alpha_{j}}\left(\theta_{j}\right)
$$

then we see that (3) can be written

$$
\int_{\left(\mathbb{R}^{d}\right)^{n}} e^{\widehat{\otimes} \alpha} d B^{\otimes|\alpha|}=H_{\alpha}(\omega)
$$

using multi-index notation: $e^{\widehat{\otimes} \alpha}=e_{1}^{\widehat{\otimes} \alpha_{1}} \widehat{\otimes} \ldots \widehat{\otimes} e_{m}^{\widehat{\otimes} \alpha_{m}}$ if $e=\left(e_{1}, e_{2}, \ldots\right)$. Since the family $\left\{e^{\widehat{\otimes} \alpha} ;|\alpha|=n\right\}$ forms an orthonormal basis for the symmetric functions in $L^{2}\left(\left(\mathbb{R}^{d}\right)^{n}\right)$, we see by combining (2) and (4) that we have the representation

$$
F(\omega)=\sum_{\alpha} c_{\alpha} H_{\alpha}(\omega)
$$

(the sum being taken over all multi-indices $\alpha$ of nonnegative integers). Moreover, it can be proved that

$$
\|F\|_{L^{2}(\mu)}^{2}=\sum_{\alpha} \alpha ! c_{\alpha}^{2}
$$

where $\alpha !=\alpha_{1} ! \ldots \alpha_{m}^{\prime} !$

The Hida test function space $(\mathcal{S})$ and the Hida distribution space $(\mathcal{S})^{*}$ can be given the following characterization, due to Zhang [Z].

Theorem 2.1: Let $\psi \in L^{2}(\mu)$ have the chaos expansion

$$
\psi(\omega)=\sum_{\alpha} c_{\alpha} H_{\alpha}(\omega)
$$


Then $\psi$ is a Hida test function, i.e. $\psi \in(\mathcal{S})$, if

$$
\sup _{\alpha} c_{\alpha}^{2} \alpha !(2 I)^{\alpha k}<\infty, \forall \text { natural numbers } k<\infty
$$

where

$$
(2 N)^{\alpha}:=\prod_{j=1}^{m}\left(2^{d} \beta_{1}^{(j)} \ldots \beta_{d}^{(j)}\right)^{\alpha_{j}} \text { for } \alpha=\left(\alpha_{1}, \ldots, \alpha_{m}\right)
$$

A Hida distibution $\Psi, \Psi \in(\mathcal{S})^{*}$, is a formal series

$$
\Psi=\sum_{\alpha} b_{\alpha} H_{\alpha}
$$

where

$$
\sup _{\alpha} b_{\alpha}^{2} \alpha !\left((2 \mathbb{N})^{-\alpha}\right)^{q}<\infty \text { for some } q>0
$$

If $\Psi \in(\mathcal{S})^{*}$ and $\psi \in(\mathcal{S})$ is given as in the theorem, the action of $\Psi$ on $\psi$ is given by

$$
\langle\langle\Psi, \psi\rangle\rangle=\sum_{\alpha} \alpha ! b_{\alpha} c_{\alpha}
$$

Note that no assumptions are made regarding the convergence of the formal series in (6). We can in a natural way regard $L^{2}(\mu)$ as a subspace of $(\mathcal{S})^{*}$. In particual, if $F \in L^{2}(\mu)$ then by $(7)$ the action of $F$ on $\psi \in(\mathcal{S})$ is given by

$$
\langle\langle F, \psi\rangle\rangle=E[F \cdot \psi]
$$

Since 1 is an element of $(\mathcal{S})$, the expectation function can be extended to $(\mathcal{S})^{*}$ :

$$
E[\Psi]=\langle\langle\Psi, 1\rangle\rangle
$$

We will now introduce the spaces $(\mathcal{S})^{1}$ and $(\mathcal{S})^{-1}$ which were first constructed by Kondratiev $[\mathrm{K}]$. For a complete account on the following results, see Albeverio et. al. [ADKS] and Kondratiev et. al. [KLS]:

Definiton 2.2: Define $(\mathcal{S})^{\rho}$ and $(\mathcal{S})^{-\rho}$ as follows:

Part a): For $0 \leq \rho \leq 1$ let $(\mathcal{S})^{\rho}$ consist of all

$$
\psi=\sum_{\alpha} c_{\alpha} H_{\alpha} \in L^{2}(\mu)
$$

such that

$$
\|\psi\|_{\rho, k}^{2}:=\sum_{\alpha} c_{\alpha}^{2}(\alpha !)^{1+\rho}(2 I N)^{\alpha k}<\infty \text { for all } k<\infty
$$


Part b): The space $(\mathcal{S})^{-\rho}$ consists of all formal expansions

$$
\Psi=\sum_{\alpha} b_{\alpha} H_{\alpha}
$$

such that

$$
\sum_{\alpha} b_{\alpha}^{2}(\alpha !)^{1-\rho}(2 \mathbb{N})^{-\alpha q}<\infty \text { for some } q<\infty
$$

The family of seminorms $\|f\|_{\rho, k}^{2} ; k=1,2, \ldots$ defines a topology on $(\mathcal{S})^{\rho}$.

We remark that $(\mathcal{S})=(\mathcal{S})^{0}$ and $(\mathcal{S})^{*}=(\mathcal{S})^{-0}$ in the above construction. $(\mathcal{S})^{-1}$ will be called the space of Kondratiev distributions.

Definition 2.3: Let $\Phi=\sum_{\alpha} a_{\alpha} H_{\alpha}, \Psi=\sum_{\alpha} b_{\beta} H_{\beta}$ be two elements of $(\mathcal{S})^{-\rho}$. Then the Wick product of $\Phi$ and $\Psi$ is the element $\Phi \diamond \Psi$ in $(\mathcal{S})^{-\rho}$ given by

$$
\Phi \diamond \Psi=\sum_{\alpha, \beta} a_{\alpha} b_{\beta} H_{\alpha+\beta}
$$

It can be shown that $(\mathcal{S})^{1}$ is closed under the Wick product.

The Hermite Transform, see Lindstrøm et. al. [LøU], has a natural extension to $(\mathcal{S})^{-1}$, the space of Kondratiev distributions:

Definition 2.4: If $\Psi=\sum_{\alpha} b_{\alpha} H_{\alpha} \in(\mathcal{S})^{-1}$ then the Hermite Transform of $F, \mathcal{H} \Psi=\tilde{\Psi}$, is defined by

$$
\tilde{\Psi}(z)=\mathcal{H} \Psi(z)=\sum_{\alpha} b_{\alpha} z^{\alpha}
$$

where $z=\left(z_{1}, \dot{z}_{2}, \ldots\right) \in \mathbb{C}_{0}^{\mathbb{N}}$, and

$$
z^{\alpha}=z_{1}^{\alpha} z_{2}^{\alpha} \ldots z_{m}^{\alpha}
$$

for $\alpha=\left(\alpha_{1}, \ldots, \alpha_{m}\right)$.

The Hermite Transform characterizes $(\mathcal{S})^{-1}$ in the following way:

Lemma 2.5: $\Psi \in(\mathcal{S})^{-1}$ if and only if there exist some $\epsilon>0, q<\infty$ such that the Hermite transform of $\Psi, \mathcal{H} \Psi$, is a bounded analytic function on $B_{q}(0, \epsilon)$.

Convergence of sequences in $(\mathcal{S})^{-1}$ can be characterized in terms of the Hermite Transform as follows:

Lemma 2.6: The following are equivalent

I: $\Psi_{n} \rightarrow \Psi$ in $(\mathcal{S})^{-1}$

II: There exist $\epsilon>0, q<\infty, M<\infty$ such that

$$
\mathcal{H} \Psi_{n}(z) \rightarrow \mathcal{H} \Psi(z) \text { as } n \rightarrow \infty \text { for } z \in \mathbf{B}_{q}(0, \epsilon)
$$


and

$$
\left|\mathcal{H} \Psi_{n}(z)\right| \leq M \text { for all } n=1,2, \ldots ; z \in \mathbf{B}_{q}(0, \epsilon)
$$

where

$$
\mathbf{B}_{q}(0, \epsilon)=\left\{z=\left(z_{1} ; z_{2}, \ldots\right) \in \mathbb{C}_{0}^{\mathbb{N}} ; \sum_{\alpha}\left|z^{\alpha}\right|^{2}(2 \mathbb{N})^{\alpha q}<\epsilon^{2}\right\}
$$

Note that the Hermite Transform transforms the Wick product into an ordinary product.

The Wick product gives a nice relation between functional integration in $(\mathcal{S})^{-1}$ and Skorohod/Ito integration. We define integration in $(\mathcal{S})^{-1}$ as föllows:

Definition 2.7: Assume $\Psi_{s} \in(\mathcal{S})^{-1}$ for each $s \in[0, T]$, where $0<T \leq \infty$. If

$$
\left\langle\left\langle\Psi_{s}, \psi\right\rangle\right\rangle \in L^{1}([0, T], d s)
$$

for all $\psi \in(\mathcal{S})^{1}$, we define the unique $(\mathcal{S})^{-1}$-element $\int_{0}^{T} \Psi_{s} d s$ by

$$
\left\langle\left\langle\int_{0}^{T} \Psi_{s} d s, \psi\right\rangle\right\rangle=\int_{0}^{T}\left\langle\left\langle\Psi_{s}, \psi\right\rangle\right\rangle d s
$$

Consider the case $d=1$, i.e the probability space $\mathcal{S}^{\prime}(\mathbb{R})$ : Define the element

$$
W_{t}=\sum_{k=1}^{\infty} \xi_{k}(t) H_{\epsilon_{k}}
$$

where $\epsilon_{k}$ is the multi-index with zeros except at position $k$, where it has value 1. It can be shown, see for instance Gjessing et. al. [GHLØUZ], that $W_{t} \in(\mathcal{S})^{*}$. Moreover, for a Skorohod integrable element $\Psi_{s} \in L^{2}(\mu)$ it can be shown that

$$
\int_{0}^{t} \Psi_{s} \diamond W_{s} d s=\int_{0}^{t} \Psi_{s} \delta B_{s}
$$

where the integral on the right hand side is the Skorohod integral. See Lindstrøm et. al. [LØU], Hida et. al. [HKPS] and Benth [B] for a discussion of this relation. We can say that functional integration in $(\mathcal{S})^{-1}$ involving Wick product with $W_{t}$ generalizes the Skorohod/Ito integration. This connection motivates the following interpretation of (1): We look for an element $X_{t}$ in $(\mathcal{S})^{-1}$ which satisfies

$$
X_{t}=X_{0}+r \int_{0}^{t} X_{s} \diamond\left(N-X_{s}\right) d s+\alpha \int_{0}^{t} X_{s} \diamond\left(N-X_{s}\right) \diamond W_{s} d s
$$

We end this section with a nice property of the $(\mathcal{S})^{-1}$ space, the so-called Wick Calculus theorem, see theorem 12 in Kondratiev et. al. [KLS]:

Theorem 2.8: Let $\Psi \in(\mathcal{S})^{-1}$. Assume $f: \mathbb{C} \rightarrow \mathbb{C}$ is analytic in a neighborhood of $E[\Psi]$. Then

$$
f(\tilde{\Psi}(z))=\tilde{\Phi}(z)
$$

is the Hermite Transform of an element $\Phi$ of $(\mathcal{S})^{-1}$.

We remark that the definitions and results presented in the above language can be found in Holden et. al. [HL $\varnothing \mathrm{UZ}$ ]. 


\section{The Solution Of The Population Modell}

For simplicity we will assume that $N=1$ in modell (8). We also assume that $X_{0} \in$ $(\mathcal{S})^{-1}$ and that

$$
E\left[X_{0}\right]>0
$$

Hermite transforming the stochastic equation (8) into an ordinary' complex differential equation, and solving, we obtain the candidate

$$
X_{t}=\left(1+\Theta_{0} \diamond \exp ^{\circ}\left(-r t-\alpha B_{t}\right)\right)^{\diamond(-1)}
$$

where

$$
\Theta_{0}=\left(1-X_{0}\right) \diamond X_{0}^{\diamond(-1)}=X_{0}^{\diamond(-1)}-1
$$

We have written $\exp ^{\diamond}$ for the Wick exponential, i.e the element defined by

$$
\exp ^{\circ} \Phi=\sum_{n=1}^{\infty} \frac{1}{n !} \Phi^{\diamond n}
$$

(see theorem 2.8 above.) We show that $X_{t}$ is an element in $(\mathcal{S})^{-1}$ :

Define

$$
g(z)=z^{-1}-1
$$

Obviously, $g(z)$ is analytic in a neighborhood around $E\left[X_{0}\right]>0$. Hence, $\Phi_{0}$ is an element of $(\mathcal{S})^{-1}$ Furthermore, define

$$
f(z)=(1+z)^{-1}
$$

We have

$$
E\left[\Phi_{0} \diamond \exp ^{\diamond}\left(-r t-\alpha B_{t}\right)\right]=\left(E\left[X_{0}\right]^{-1}-1\right) e^{-r t}
$$

When $0<E\left[X_{0}\right] \leq 1$ we have

$$
0 \leq\left(E\left[X_{0}\right]^{-1}-1\right) e^{-r t} \leq\left(E\left[X_{0}\right]^{-1}-1\right)
$$

and, when $E\left[X_{0}\right]>1$,

$$
\left(E\left[X_{0}\right]^{-1}-1\right) \leq\left(E\left[X_{0}\right]^{-1}-1\right) e^{-r t}<0
$$

for all $t \geq 0$. In both cases is the expectation bounded away from -1 for all $t \geq 0$. Hence, there exist constants $q, \epsilon$ such that

$$
f\left(\tilde{\Phi_{0}}(z) \exp \left(-r t-\alpha \tilde{B}_{t}(z)\right)\right)
$$

is analytic and bounded for $z \in B_{q}(0, \epsilon)$, for all $t \geq 0$. This implies by theorem 2.8 that $X_{t}$ is an element of $(\mathcal{S})^{-1}$ for all $t \geq 0$.

To show that $X_{t}$ is a solution of equation (8), we must prove that $X_{t}^{\prime}$ satisfies

$$
\frac{d X_{t}}{d t}=\left(r+\alpha W_{t}\right)^{\prime} \diamond X_{t} \diamond\left(1-X_{t}\right)
$$


in $(\mathcal{S})^{-1}$. But by lemma 2.6 , II this is equivalent with showing that

$$
\frac{\tilde{X}_{t+h}(z)-\tilde{X}_{t}(z)}{h} \rightarrow\left(r+\alpha \tilde{W}_{t}(z)\right) \tilde{X}_{t}(z)\left(1-\tilde{X}_{t}(z)\right)
$$

pointwise boundedly for $z \in B_{q}(0, \epsilon)$ when $h \rightarrow 0$. 'This can be seen to hold by direct calculation: The Hermite transform of $(9)$ is:

$$
\tilde{X}_{t}(z)=\left(1+\tilde{\Theta}_{0}(z) e^{-r t-\alpha \tilde{B}_{t}(z)}\right)^{-1}
$$

Hence, after some manipulation,

$$
\begin{aligned}
\frac{\tilde{X}_{t+h}(z)-\tilde{X}_{t}(z)}{h}= & \frac{\left(\frac{1-e^{-r h}}{h} e^{-\alpha \tilde{B}_{t}(z)}+e^{-r h} \frac{\left(e^{-\alpha \tilde{B}_{t}(z)}-e^{-\alpha \tilde{B}_{t+h}(z)}\right)}{h}\right)}{h} \\
& \times e^{-r t} \tilde{\Theta}_{0}(z) \tilde{X}_{t}(z) \tilde{X}_{t+h}(z)
\end{aligned}
$$

We see that $\tilde{X}_{t+h}(z) \rightarrow \tilde{X}_{t}(z)$ pointwise boundedly for $z \in \mathbf{B}_{q}(0, \epsilon)$. By definition we have

$$
\frac{d}{d t} e^{-\alpha \tilde{B}_{t}(z)}=\lim _{h \rightarrow 0} \frac{e^{-\alpha \tilde{B}_{t}(z)}-e^{-\alpha \tilde{B}_{t}(z)}}{h}=\alpha \tilde{W}_{t}(z) e^{-\alpha \tilde{B}_{t}(z)}
$$

for every $z \in \mathbf{B}_{q}(0, \epsilon)$. Moreover, we can show that this convergence is bounded on $\mathbf{B}_{q}(0, \epsilon)$. Hence

$$
\frac{\tilde{X}_{t+h}(z)-\tilde{X}_{t}(z)}{h} \rightarrow\left(r+\alpha \tilde{W}_{t}(z)\right) \tilde{X}_{t}(z)\left(1-\tilde{X}_{t}(z)\right)
$$

pointwise boundedly on $\mathbf{B}_{q}(0, \epsilon)$.

Since $\tilde{X}_{t}(z)$ is the unique solution of the Hermite transformed version of equation (8), it follows by injectivity of the Hermite transform that $X_{t}$ is unique. We have the conclusion:

Theorem 3.1: Assume $X_{0} \in(\mathcal{S})^{-1}$ with $E\left[X_{0}\right]>0$. Then

$$
X_{t}=\left(1+\Theta_{0} \diamond \exp ^{\diamond}\left(-r t-\alpha B_{t}\right)\right)^{\diamond(-1)}
$$

where

$$
\Theta_{0}=X_{0}^{\circ(-1)}-1
$$

is the unique $(\mathcal{S})^{-1}$ solution of $(8)$ with $N=1$. 


\section{Some Concluding Remarks}

As pointed out in the introduction, Lindstrøm et. al. [LøU] did not obtain any solution of (8) for the "stochastic bifurcation point" $X_{0}=\frac{1}{2}$. We discuss this special case more in detail: With initial condition $X_{0}=\frac{1}{2}$, we obtain $\Theta_{0}=1$ which gives the solution

$$
X_{t}=\left(1+\exp ^{\diamond}\left(-r t-\alpha B_{t}\right)\right)^{\diamond(-1)}
$$

We show that $X_{t}$ is not an element of the Hida distribution space $(\mathcal{S})^{*}$ : In Hida et. al. [HKPS] the S-transform of an element of $(\mathcal{S})^{*}$, is defined as

$$
S F(\xi)=\left\langle\left\langle F, \exp ^{\diamond} W_{\xi}\right\rangle\right\rangle
$$

for $\xi \in \mathcal{S}(\mathbb{R})$. The S-transform of $X_{t}$ given in (11), is

$$
S X_{t}(\xi)=\left(1+\exp \left(r t+\alpha \int_{0}^{t} \xi(s) d s\right)\right)^{-1}
$$

This object is well defined for all $\xi \in \mathcal{S}(\mathbb{R})$, and $v_{t}=S X_{t}(\xi)$ is the unique solution of the problem

$$
v_{t}=\frac{1}{2}+r \int_{0}^{t} v_{s}\left(1-v_{s}\right) d s+\alpha \int_{0}^{t} v_{s}\left(1-v_{s}\right) \xi(s) d s
$$

for each $\xi$. However, $S X_{t}(\xi)$ can not be extended to an analytic function

$$
z \rightarrow S X_{t}(z \xi)
$$

on the complex plane $\mathbb{C}$. Hence, $X_{t}$ is not an element of $(\mathcal{S})^{*}$. (See Hida et. al. [HKPS] for a characterization of $(\mathcal{S})^{*}$-elements in terms of the S-transform. We see that for $\xi, \eta \in \mathcal{S}(\mathbb{R})$ the mapping.

$$
\lambda \rightarrow S X_{t}(\xi+\lambda \eta)
$$

can only be analytic in a neighborhood of zero in $\mathbb{C}$. This tells us that $X_{t}$ is not contained in any of the spaces $(\mathcal{S})^{-\rho}, \rho \in[0,1)$. (See Albeverio et. al. [ADKS] for the characterization of these spaces by the S-transform.)

By the uniqueness of the Hermite Transform, the $(\mathcal{S})^{-1}$ element $X_{t}$ given in $(9)$ and (10) has to coincide with the solution found by Lindstrøm et. al. [LøU] for constant initial conditions $X_{0}=x \neq \frac{1}{2}$. As we have seen, the results above are worked out for general initial conditions

$$
X_{0} \in(\mathcal{S})^{-1}
$$

where $E\left[X_{0}\right]>0$. This means that for stochastic variables as initial conditions we have a solution as well. Note that the case of anticipating initial conditions is also included.

Acknowledgements: This work has been supported by the Norwegian Research Council(NFR) under grant (NAVF)100549/410. The author would like to thank professor Bernt 
